\title{
Perméabilité des digues en enrochements aux ondes de gravité périodiques*
}

\author{
The perviousness of rock fill breakwaters \\ to periodic gravity waves *
}

(SUITE ET FIN)

\author{
(Cf. La Houille Blanche, $\mathrm{n}^{\circ} 6,1957$ ) \\ PAR B. LE MÉHAUTÉ \\ INGÉNIEUR E.N.S.E.H.T. - INGÉNIEUR a LA SOGREAH \\ (Actuellement Professeur Adjoint à l'Ecole Polytechnique de Montréal)
}

\begin{abstract}
AVANT-PROPOS et Notations.
Première partie. - Etude des lols de l'́́couLEMENT PÉROOMUUE DANS UN MASSIF PERMÉatBLE.

Chap. I. Equations dil moubement.

Chap. II. Intégration de l'équation générale du mollvement.

Chap. IIr. Perméabilité d'un massif long soumis à une onde incidente.

Chap. IV. Massif de largeur faible par rapport $\dot{a}$ la'longueur d'onde. .

DeUxième partie. - Etude DEs LoIs DE SIMilyTUDE DE LA PERMÉabILTTÉ D'UN MASSIF EN ENROCHEMENTS.

Chap. I. Position du problème. Difficultess d'une semblable étude.

Chap. II. Etablissement des règles de similitude de l'écoulement à travers un massif perméable.

Chap. III. Similitude des écoulements dans un massif en enrochements en régime permanent. Chap. IV. Possibilité d'application des régles de similitude.

Chap. V. Applications pratiques aux digues en enrochements.

Remarques.

Thoisième partie. - Recherches ExpérmienTALES.

Chap. I. Appareillage expérimental: 1) Canaux a houle. 2) Appareillage de mesure. 3) Les mesures des caractéristiques du massif.

Chap. II. Résultats expérimentaux obtenus avec des massifs de largenr constante.

Chap. III. Description physique des phénomines et examen des résultats expérimentaux.

Chap. IV. Vérification des lois de similitude.

Chap. V. Calculs de profits de digues en enrochements et résultats d'essais.

Chap. VI. Précision des résultats : 1) ErreuI sur la connaissance des lois de l'écoulement. 2) Erreurs expérimentales. 3) Erreurs sur la connaissance des conditions naturelles. Conclusion.
\end{abstract}

Preface and Headings.

Part 1. StUdy of THE PERIOdic Flow laws in a PERMEABLE STRUCTURE.

Chapter I. Equations of movement.

Chapter II. Integration of the general equation of movement.

Chapter IIr. Permeability of an extended structure subjected to an incident wave.

Chapter IV. Small width structure in relation to wave length.

PaRt 2. STUdy of The SIMILITUde LaWs FOR PEBMEABILITY IN A ROCK FILLED STRUCTURE.

Chapter I. Posing the problem. Difficulties of a similarity study.

Chapter Ir. Establishing the rules for flow similarity throngh a permeable structure.

Chapter III. Flow similitude in a rock filled structure in permanent regime.

Chapter IV. Possible application of similitude laws.

Chapter V. Practical application to rock fill dykes.

Comments.

Part 3. experimental research.

Chapter I. Experimental equipment : a) Wave flumes. b) Instrument equipment. c) Measuring the characteristics of the structure.

Chapter II. Results obtained from tests on constant width structures.

Chapter III. Physical description of the phenomena and analysis of test results.

Chapter IV. Verification of the similitude laws. Chapter V. Calculation of rock fill dyke profiles and test results.

Chapter VI. Accuracy of results : a) Error associated with the flow laws. b) Experimenal errors. c) Error from uncertain knowledge of the true life conditions.

Conclusion.

* Thèse présentée devant la Faculté des Sciences de l'Université de Grenoble pour l'obtention du titre d'Ingénieur-Docteur. 


\title{
TROISIEME PARTIE
}

\section{RECHERCHES EXPERIMENTALES}

\author{
CHAPITRE V
}

\section{CALCULS DE PROFILS DE DIGUES EN ENROCHEMENTS ET RÉSULTATS D'ESSAIS}

Le principe du calcul du profil répondant aux lois de similitude de perméabilité a été exposé dans la deuxième partie de notre ouvrage. Avant de passer à son application, nous préciserons certains points.

\section{L'INDICE DE VIDE.}

Faute de renseignements sur la valeur exacte des indices de vide des matériaux destinés à construire la jetée, nous avons pris les valeurs suivantes pour les cas particuliers traités ici.

\begin{tabular}{|c|c|}
\hline $\begin{array}{c}\text { Prowi. } \\
\text { en enrochements }\end{array}$ & $\begin{array}{l}\text { Profir } \\
\text { avec blocs à contre-pente }\end{array}$ \\
\hline $\mathrm{TV}<500 \mathrm{~kg} \ldots \ldots 30 \%$ & $\mathrm{TV}<200 \mathrm{~kg} \ldots \ldots 32 \%$ \\
\hline $500 \mathrm{~kg}$ à $2 \mathrm{t} \ldots \ldots 35 \%$ & $200 \mathrm{~kg}$ à $2 \mathrm{t} \ldots \ldots 32 \%$ \\
\hline $2 \mathrm{t}$ à $10 \mathrm{t} \ldots \ldots 35 \%$ & $2 t$ a $5 \mathrm{t}$. \\
\hline $10 \mathrm{t}$ à $20 \mathrm{t} \ldots \ldots .40 \%$ & $5 \mathrm{t}$ à $15 \mathrm{t} \ldots \ldots \ldots 38 \%$ \\
\hline
\end{tabular}

\section{Diamètres caractéristigues} DES ENROCHEMENTS.

Nous avons vu que les diamètres caractéristiques auxquels les calculs doivent se référer correspondent aux granulométries les plus petites pour chaque zone considérée. Nous baserons done nos calculs sur les poids des matériaux les plus fins, en assimilant cenx-ci à des sphères de même poids.
Si nous supposons que la densité des enrochements est de 2,8, le diamètre sera donné par la formule :

$$
d=\sqrt[3]{\frac{6 \mathrm{P}}{2,8 \pi}}
$$

Pour le tout-venant des jetées entièrement construites en enrochements, nous avons pris un poids minimum de $10 \mathrm{~kg}$ correspondant sensiblement à un diamètre de $20 \mathrm{~cm}$, pour les jetées en enrochements. Pour les jetées avec blocs à contre-pente, nous avons pris un poids minimum de $5 \mathrm{~kg}$ correspondant à un diamètre de $16 \mathrm{~cm}$.

Le calcul du profil sera donc fait pour chaque zone granulométrique en se référant au diamètre des enrochements de poids minimum. Les coefficients de grossissement $K$ ainsi calculés serviront à déterminer les granulométries de toute la couche considérée. Les enrochements les plus gros auront donc le même grossissement que les enrochements les plus petits de la même couche. Par conséquent, mélangés dans une proportion identique à celle de la nature, ils doivent théoriquement assurer le même indice de vide. On concoit que cette considération théorique nécessite, en premier lieu, un travail de triage d'enrochements long et délicat puisque l'on cherche à obtenir un indice de vide bien déterminé. Ce travail doit être fait par approximations successives, guidé par exemple par les diagrammes ternaires de Ferret. Mais la mesure de l'indice de vide étant particulièrement influencée par le tassement et par les effets des parois, nous n'avons pu obtenir une précision supérieure à $2 \%$. 
PROFILS CALCULÉS ET ESSAIS EFFECTUÉS

\begin{tabular}{|c|c|c|c|}
\hline Profil & $\begin{array}{l}\text { Similitude } \\
\text { respectée } \\
\text { pour } 2 a=\end{array}$ & $\begin{array}{c}\text { Niveau } \\
\Delta \mathrm{Hm}\end{array}$ & $\begin{array}{l}\text { Périodes } \\
\text { essayées } \\
\text { en s }\end{array}$ \\
\hline Profil en enrochements avec masque étanche......... (1) & $1 \mathrm{~m}$ & 0,00 & $10,20,30,35$ \\
\hline \multirow{2}{*}{ Profil en enrochements sans masque étanche......... (2) } & \multirow{2}{*}{$1 \mathrm{~m}$} & 0,00 & $10,20,30,35$ \\
\hline & & 2,00 & 35 \\
\hline Profil avec blocs à contre-pente non jointifs.......... (3) & $1 \mathrm{~m}$ & 0,00 & $10,20,30,35$ \\
\hline Profil avec blocs à contre-pente jointifs. . . . . . . . . (3) & $1 \mathrm{~m}$ & 0,00 & 35 \\
\hline \multirow{2}{*}{ Profil en enrochements avec masque étanche.......... (4) } & \multirow{2}{*}{$10 \mathrm{~m}$} & 0,00 & $10,20,30,35$ \\
\hline & & 2,00 & 35 \\
\hline
\end{tabular}

Nous avons vu comment on détermine la valeur $l$ des ouvrages par la formule :

$$
l=l_{i}=\Sigma l_{j}\left(d_{i} / d_{j}\right)^{n}
$$

Nous considérerons que dans la nature l'écoulement est toujours turbulent et nous prendrons par suite $n=1$.

Le premier travail consiste à tracer sur le plan des ouvrages projetés les lignes de courant les plus probables. Nous verrons qu'une erreur sensible sur ce tracé se traduit par une erreur très faible sur les coefficients de grossissement. Il est donc tout à fait inutile de chercher une grande précision dans le tracé de ces lignes.

Le calcul de la longueur $I$ étant fait pour chaque courbe granulométrique, on détermine le groupement de variables $\mathrm{H}_{e} d^{3} \varepsilon^{5} / l$ qui, à l'aide de l'abaque $n^{\circ} 6$, nous donne le coefficient de grossissement $K$ en fonction de l'échelle. Nous avons pris une charge efficace respectivement égale à $1 \mathrm{~m}$ et à $10 \mathrm{~m}$.

Lorsque la charge efficace est de $1 \mathrm{~m}$, on peut considérer que les résultats trouvés sur le modèle sont en similitude pour la transmission des houles de beau temps ou pour la transmission d'ondes longues auxquelles se superposent ces houles.

Lorsque la charge efficace est de $10 \mathrm{~m}$, on peut considérer que les résultats obtenus correspondent pratiquement à la transmission des houles de tempête ou à la transmission d'ondes longues pendant une tempête.
Un premier tableau ci-dessus donne les conditions d'essais: sur les tableaux suivants, nous avons détaillé les différents calculs nous donnant les granulométries minimum et maximum du modèle.

Dans une première colonne, nous donnons la catégorie des enrochements constituant les digues, puis le poids minimum et maximum de ces catégories et les granulométries correspondantes.

Dans une quatrième colonne, nous trouvons l'indice de vide correspondant à chacune de ces catégories : la largeur $l$ est calculée suivant la méthode que nous avons indiquée ci-dessus. Nous donnons : tout d'abord la largeur mesurée sur les figures des digues essayées pour chacune des couches étudiées. Puis la largeur calculée en additionnant cette largeur ainsi mesurée aux largeurs des couches voisines, multipliées par le rapport des granulométries. Enfin, la largeur adoptée pour nos calculs, en faisant une moyenne lorsque les résultats obtenus dans le calcul des largeur's ne justifient pas l'adoption de plusieurs coefficients de grossissement.

Les deux colonnes suivantes donnent la valeur du produit $\mathrm{H}_{e} d^{3} \mathrm{~s}^{5} / l$ et le coefficient $\mathrm{K}$ correspondant; en multipliant ces coefficients par les granulométries minimum et maximum données dans la troisième colonne, nous obtenons les granulométries minimum et maximum du modèle pour chacune des couches granulométriques.

Les figures 18, 19 et 20 ci-après donnent 


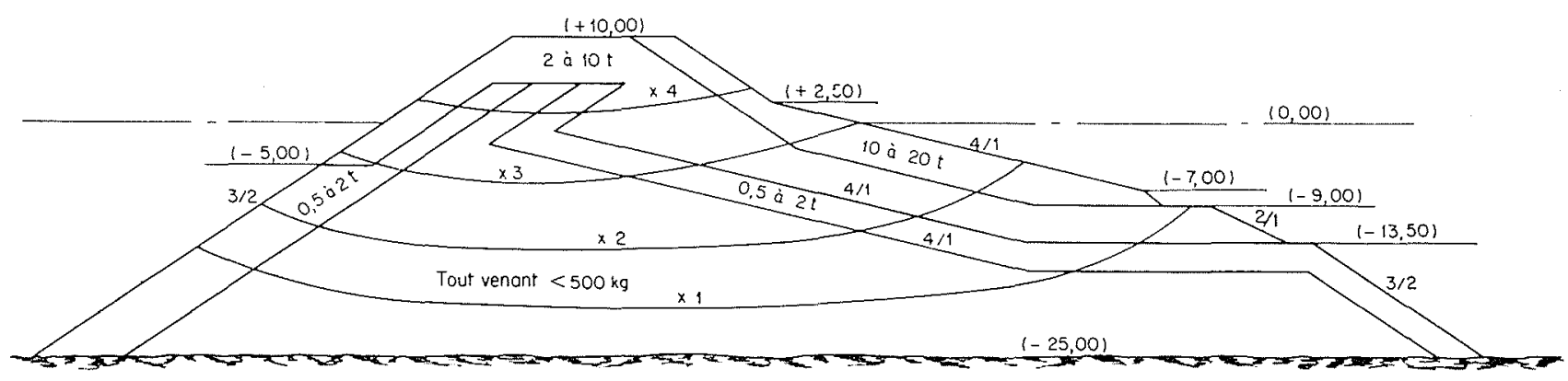

c $\begin{array}{llll}5 & 10 & 15 & 20 \mathrm{~m}\end{array}$

Fidi. 18. - Parcours moyen des trajectoires fluides à travers une digue en enrochements avec noyau étanche.

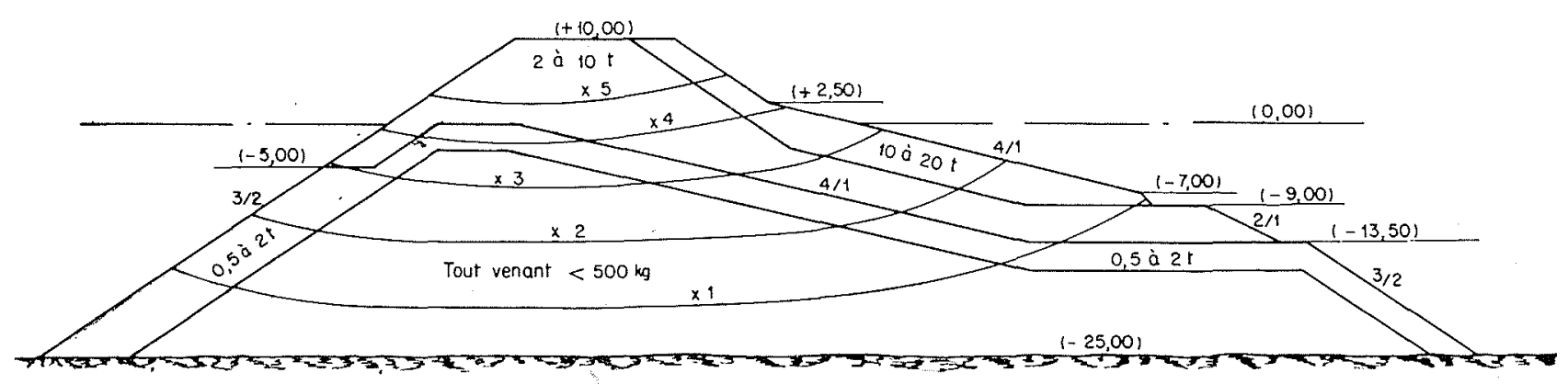

\section{$5 \quad 5 \quad 10 \quad 15 \quad 20 \mathrm{~m}$}

Fig. 19. - Parcours moyen des trajectoires fluides à travers une digue en enrochements sans noyau etanche.

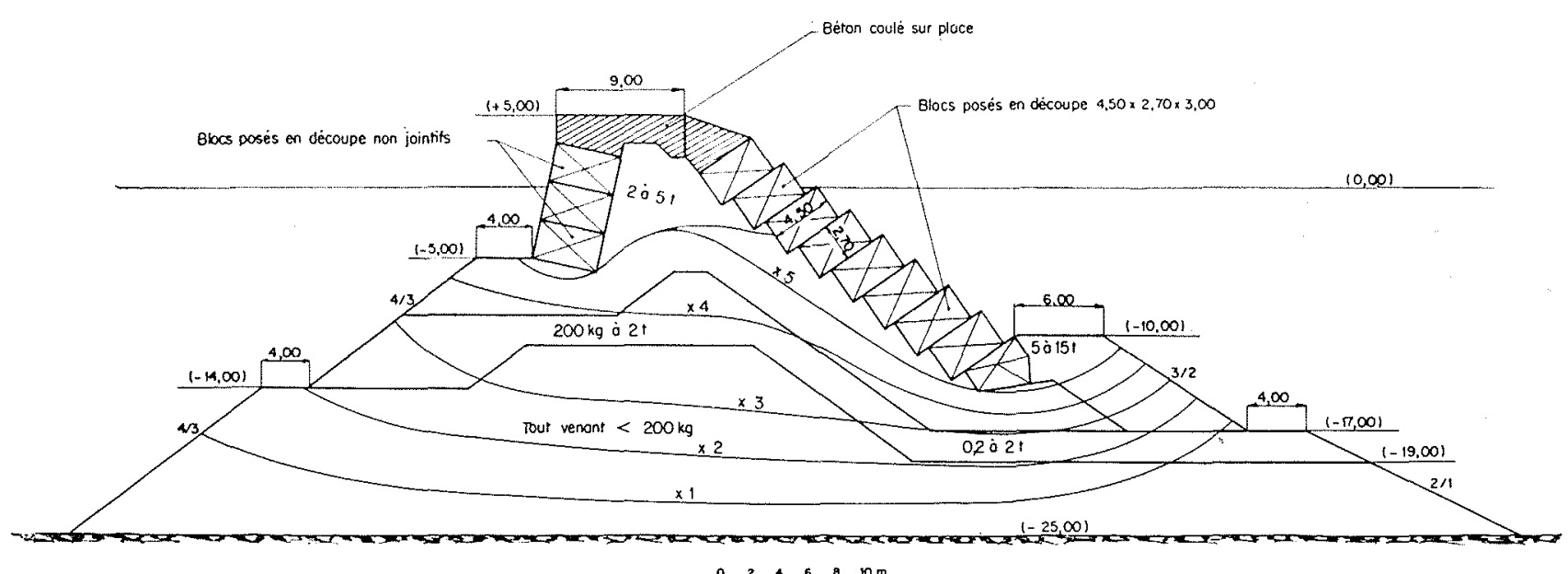

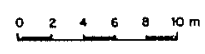

Fic. 20. - Parcours moyen des trajectoires fluides a travers une digue en blocs à contre-pente

approximativement les parcours moyens des trajectoires fluides à travers les digues.

Les quatre tableaux qui suivent domnent le détail des calculs, dont les résultats sont présentés par les figures $21-21 a-22-22 a-23-$ $23 a-24$.

Tous ces essais ont été effectués à l'échelle du $1 / 100$ dans un canal à houle de $60 \mathrm{~cm}$ de largeur et de $30 \mathrm{~m}$ de long. Le générateur d'ondes peut réaliser des périodes allant de 5 à 35 secondes nature. Les mesures des amplitudes, les clapotis partiels incidents ont été effectués à l'E.G.H. et les amplitudes transmises derrière le massif, qui sont en général très faibles, ont été 
PROFIL $\mathrm{N}^{\circ} 1$

En enrochemexts avec mascue ÉtAnche. -- Simidrude Respectée poun $\mathrm{C}=2 a=1 \mathrm{~m}$

Echelle $1 / 100$

\begin{tabular}{|c|c|c|c|c|c|c|c|c|c|c|c|c|}
\hline \multirow{2}{*}{ Catégorie } & \multicolumn{2}{|c|}{$\begin{array}{c}\text { Poids } \mathrm{kg} \\
\text { densité } 2,8\end{array}$} & \multicolumn{2}{|c|}{$\begin{array}{c}\text { Granulométrie } \\
\delta \mathrm{cm}\end{array}$} & \multirow{2}{*}{$\begin{array}{l}\text { Indice } \\
\text { de } \\
\text { vide } \\
\varepsilon \%\end{array}$} & \multicolumn{3}{|c|}{ Largetur $x \mathrm{~cm}$} & \multirow{2}{*}{$\operatorname{Cos} \varepsilon^{5} / x$} & \multirow{2}{*}{ li } & \multicolumn{2}{|c|}{$\delta_{\mathrm{MI}} \mathrm{cm}$} \\
\hline & Min. & Max. & Min. & Max. & & Mesurée & Calculée & Adoptéc & & & Min. & Max. \\
\hline \multirow{2}{*}{$<500 \mathrm{~kg}$} & \multirow{2}{*}{10} & \multirow{2}{*}{500} & \multirow{2}{*}{20} & \multirow{2}{*}{74} & \multirow{2}{*}{30} & $\begin{array}{l}x_{1}=8.750 \\
x_{2}=6.100\end{array}$ & & 7.000 & 0,30 & 8,2 & 1,7 & 6,3 \\
\hline & & & & & & $\begin{array}{l}x_{3}=2.800 \\
x_{4}=500\end{array}$ & & & & & & \\
\hline \multirow{2}{*}{$\begin{array}{c}<500 \operatorname{lgg} \\
\grave{a} \\
2 \mathrm{t}\end{array}$} & \multirow{2}{*}{500} & \multirow{2}{*}{2.000} & \multirow{2}{*}{74} & \multirow{2}{*}{117} & \multirow{2}{*}{35} & $\begin{array}{l}x_{1}=1.500 \\
x_{2}=1.500\end{array}$ & $1.500+7.000 \times(74 / 20)$ & 27.400 & 7,36 & 4,6 & 3,4 & 5,3 \\
\hline & & & & & & $\begin{array}{l}x_{3}=1.800 \\
x_{4}=1.300\end{array}$ & $1.300+1.200 \times(74 / 20)$ & 5.740 & 36,2 & 3,5 & 2,55 & 4,1 \\
\hline \multirow{2}{*}{$\begin{array}{l}10 \mathrm{t} \\
\dot{a} \\
2 \mathrm{t}\end{array}$} & \multirow[t]{2}{*}{2.000} & \multirow[t]{2}{*}{10.000} & \multirow[t]{2}{*}{117} & \multirow[t]{2}{*}{200} & \multirow{2}{*}{3,5} & $\begin{array}{l}x_{1}=1.000 \\
x_{2}=700\end{array}$ & $850+7.000 \times(117 / 20)$ & 41.750 & 20,2 & 3,9 & 4,56 & 8 \\
\hline & & & & & & $\begin{array}{l}x_{3}=900 \\
x_{4}=2.500\end{array}$ & $1.700+1.200 \times(117 / 20)$ & 8.700 & 96 & 3 & 3,5 & 6 \\
\hline \multirow{2}{*}{$\begin{array}{c}10 t \\
\dot{a} \\
20 t\end{array}$} & \multirow{2}{*}{10.000} & \multirow{2}{*}{20.000} & \multirow{2}{*}{200} & \multirow{2}{*}{252} & \multirow{2}{*}{40} & $\begin{array}{l}x_{1}=0 \\
x_{2}=600\end{array}$ & $300+7.000 \times(200 / 20)$ & 70.300 & 114 & 2,8 & 5,6 & 7,3 \\
\hline & & & & & & $\begin{array}{l}x_{3}=600 \\
x_{1}=400\end{array}$ & $500+1.200 \times(200 / 20)$ & 12.500 & 640 & 2,2 & 4,4 & 5,5 \\
\hline
\end{tabular}

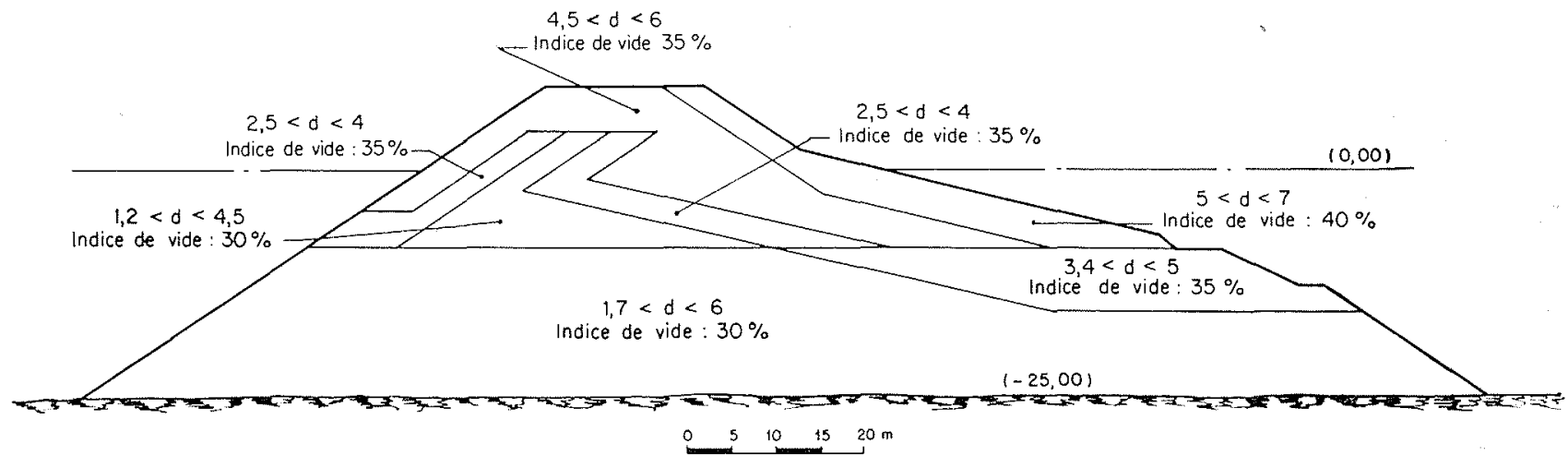

Fig. 21. - Plan du modèle réduit au 1/100 d'une digue en enrochements avec noyau étanche, la similitude étant respectée pour $2 a=1 \mathrm{~m}$. 


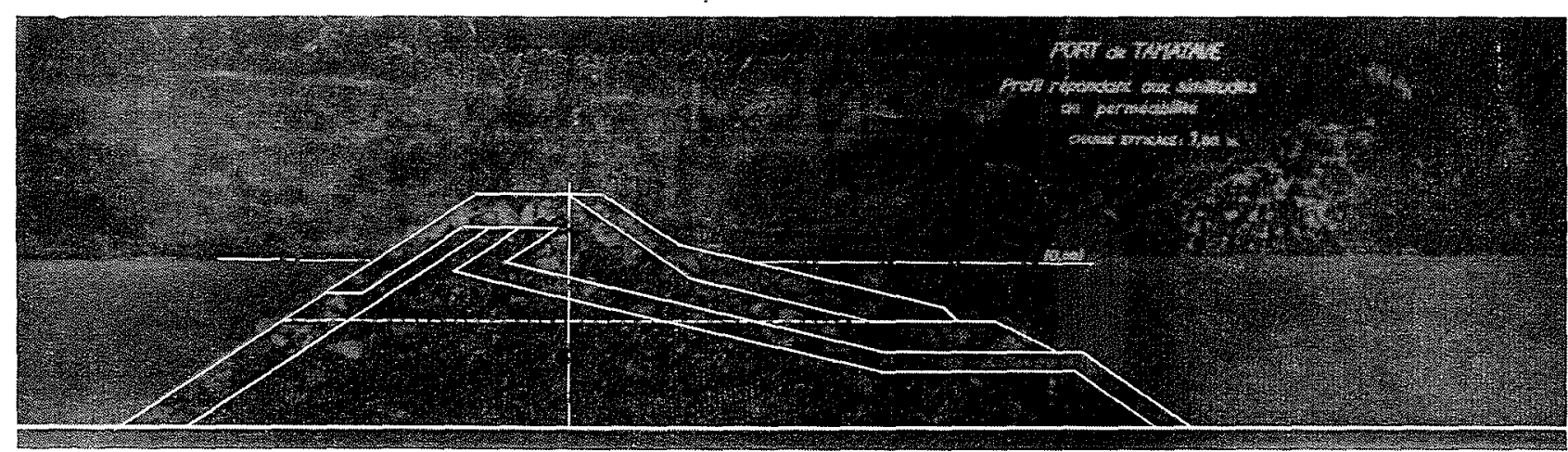

Fig. 21 a. - Photographie correspondant à la figure 21.

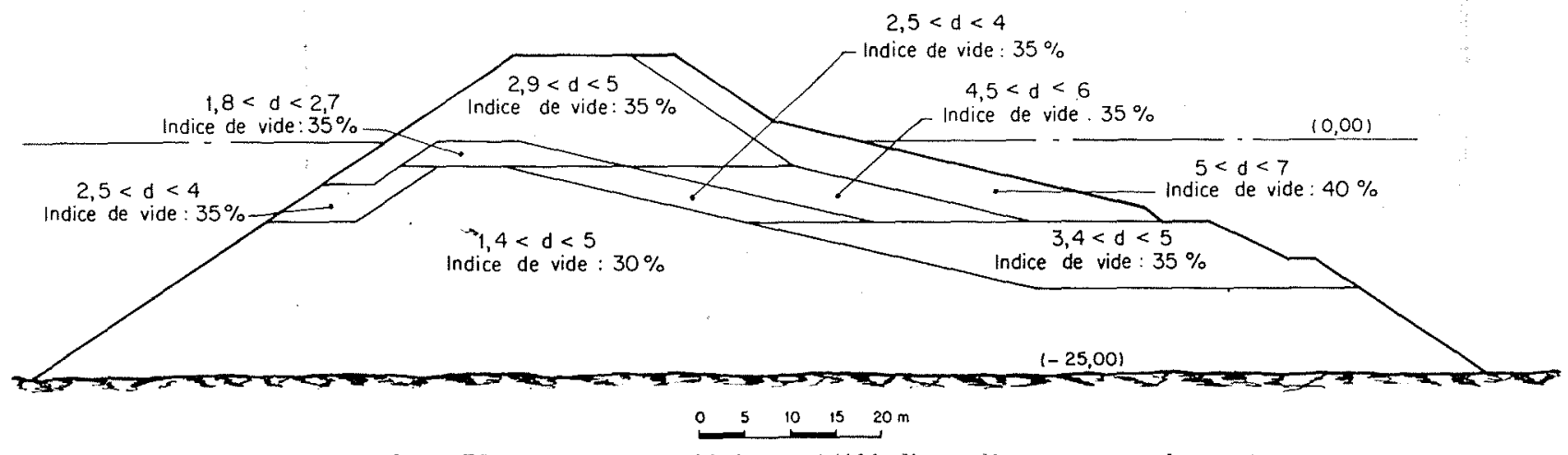

Fıg. 22. - Plan du modèle réduit au $1 / 100$ d'une digue en enrochements sans masque étanche, la similitude étant respectée pour $2 a=1 \mathrm{~m}$.

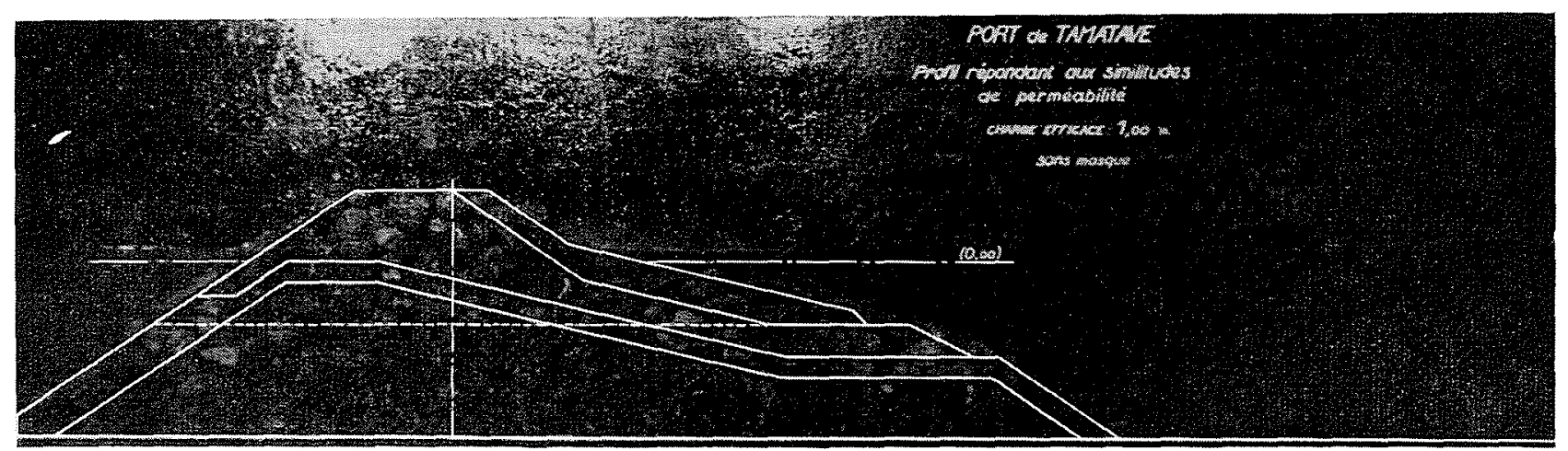

Fic. 22 a. - Photographic correspondant a la figure 22.

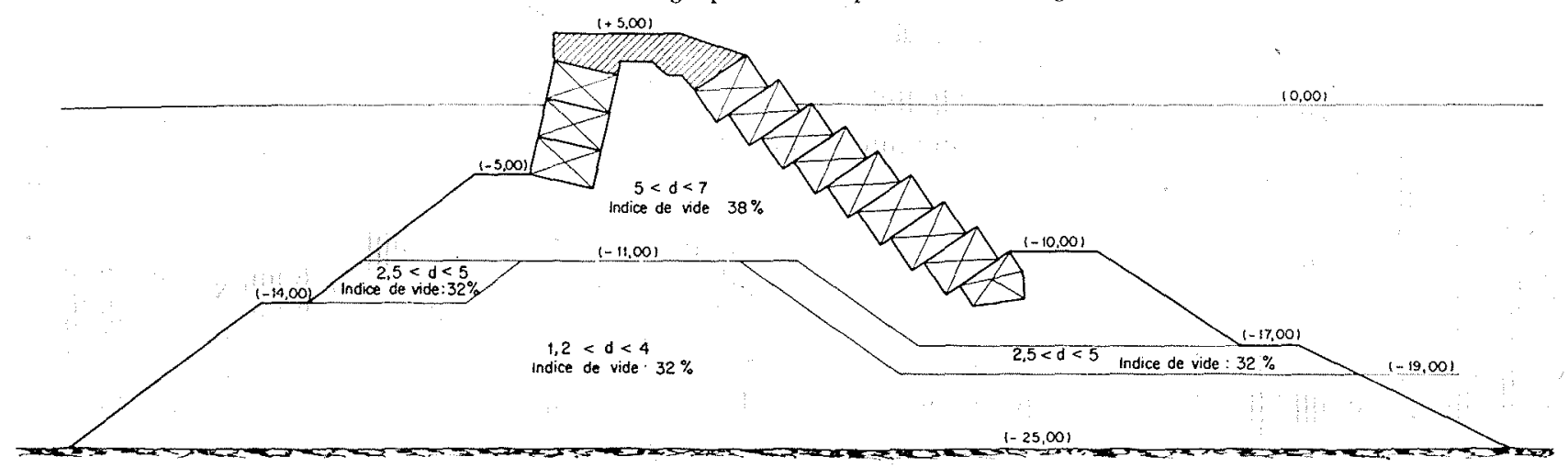

$$
\underbrace{\circ-2.510 m}
$$

Fic. 23. - Plan du modèle réduit au $1 / 100$ d'une digue avec blocs à contre-pente,

la similitude étant respectée pour $2 a=1 \mathrm{~m}$. 
PROFIL N'2

Profll en enrochements sans masque Étanche. - Similitude calculfie pour $\mathrm{C}=2 a=1 \mathrm{~m}$ Echelle 1/100

\begin{tabular}{|c|c|c|c|c|c|c|c|c|c|c|c|c|}
\hline \multirow{2}{*}{ Catégorie } & \multicolumn{2}{|c|}{$\begin{array}{r}\text { Poids kg } \\
\text { densité } 2,8\end{array}$} & \multicolumn{2}{|c|}{$\begin{array}{l}\text { Granulométrie } \\
\delta x \mathrm{~cm}\end{array}$} & \multirow{2}{*}{$\begin{array}{c}\text { Indice } \\
\text { de } \\
\text { vide } \\
\varepsilon \%\end{array}$} & \multicolumn{3}{|c|}{ Largeur $x \mathrm{~cm}$} & \multirow{2}{*}{$\operatorname{Cos}^{3} \varepsilon^{7} / x$} & \multirow{2}{*}{$\mathrm{K}$} & \multicolumn{2}{|c|}{$\delta_{\mathrm{NI}} \mathrm{cm}$} \\
\hline & Min. & Max. & Min. & Max. & & Mesurée & Calculée & Adoptée & & & Min. & Max. \\
\hline \multirow{2}{*}{$<500 \mathrm{~kg}$} & \multirow{2}{*}{10} & \multirow{2}{*}{500} & \multirow{2}{*}{20} & \multirow{2}{*}{74} & \multirow{2}{*}{30} & $\begin{array}{l}x_{1}=8.750 \\
x_{2}=6.100\end{array}$ & & 7.000 & 0,30 & 8,2 & 1,64 & 6,06 \\
\hline & & & & & & $x_{3}=2.800$ & & 2.800 & 0,70 & 7 & 1,40 & 5,18 \\
\hline \multirow{3}{*}{$\begin{array}{c}500 \mathrm{~kg} \\
\dot{\mathrm{a}} \\
2 \mathrm{t}\end{array}$} & \multirow{3}{*}{500} & \multirow{3}{*}{2.000} & \multirow{3}{*}{74} & \multirow{3}{*}{117} & \multirow{3}{*}{35} & $\begin{array}{l}x_{1}=1.500 \\
x_{2}=1.500\end{array}$ & $1.500+7.000 \times(74 / 20)$ & & 7,66 & 4,7 & 3,5 & 5,5 \\
\hline & & & & & & $x_{3}=1.800$ & $1.800+2.800 \times(74 / 20)$ & 13.000 & $\overline{16,10}$ & 3,2 & 2,3 & 3,7 \\
\hline & & & & & & $x_{4}=300$ & & 500 & 400 & 2,4 & 1,8 & 2,7 \\
\hline \multirow{3}{*}{$\begin{array}{l}2 \mathrm{t} \\
\dot{\mathrm{a}} \\
10 \mathrm{t}\end{array}$} & \multirow{3}{*}{2.000} & \multirow{3}{*}{10.000} & \multirow{3}{*}{117} & \multirow{3}{*}{200} & \multirow{3}{*}{35} & $\begin{array}{l}x_{1}=1.000 \\
x_{2}=7.000\end{array}$ & $850+7.000 \times(117 / 20)$ & 41.150 & 20,20 & 3,9 & 4,56 & 7,8 \\
\hline & & & & & & $\begin{array}{l}x_{3}=1.000 \\
x_{4}=4.660\end{array}$ & $3.300+2.800 \times(117 / 20)$ & 22.800 & 36,80 & 3,5 & 4,10 & 7 \\
\hline & & & & & & $x_{5}=3.000$ & & 3.000 & 280 & 2,5 & 2,90 & 5 \\
\hline \multirow{3}{*}{$\begin{array}{l}2 \mathrm{t} \\
\mathrm{a} \\
10 \mathrm{t}\end{array}$} & \multirow{3}{*}{10.000} & \multirow{3}{*}{20.000} & \multirow{3}{*}{200} & \multirow{3}{*}{252} & \multirow{3}{*}{40} & $\begin{array}{rr}x_{1}= & 0 \\
x_{2}= & 600\end{array}$ & $300+7.000 \times(200 / 20)$ & 70.300 & 113 & 2,8 & \multirow{2}{*}{5,6} & \multirow{2}{*}{7} \\
\hline & & & & & & $\begin{array}{l}x_{3}=600 \\
x_{4}=500 \\
\end{array}$ & $600+$ & 44.660 & 179 & 2,7 & & \\
\hline & & & & & & $x_{5}=500$ & $500+3.000 \times(200 / 117)$ & 5.600 & 142,8 & 1,8 & 3,6 & 4,5 \\
\hline
\end{tabular}

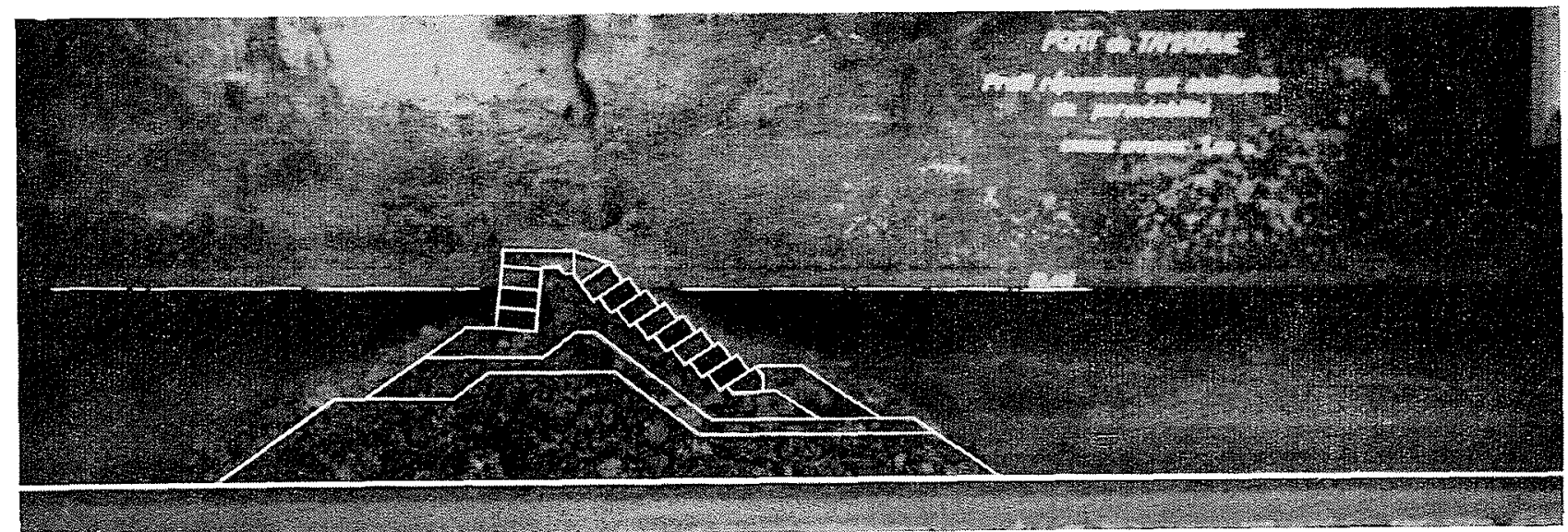

Fic. 23 a. - Photographie correspondant à la figure 23. 
PROFIL N $N^{\circ} 3$

Profil avec blocs a contre-pentes. - Similitude calculée pour $C=2 a=1 \mathrm{~m}$ Echelle 1/100

\begin{tabular}{|c|c|c|c|c|c|c|c|c|c|c|c|c|}
\hline \multirow{2}{*}{ Catégorie } & \multicolumn{2}{|c|}{$\begin{array}{l}\text { Poids } \mathrm{kg} \\
\text { densite } 2,8\end{array}$} & \multicolumn{2}{|c|}{$\begin{array}{c}\text { Granulométrie } \\
8 \mathrm{~cm}\end{array}$} & \multirow{2}{*}{$\begin{array}{l}\text { Indice } \\
\text { de } \\
\text { vide } \\
\varepsilon \%\end{array}$} & \multicolumn{3}{|c|}{ Largeur $x \mathrm{~cm}$} & \multirow{2}{*}{$\operatorname{Cog}^{3} \varepsilon^{5} / x$} & \multirow{2}{*}{$\mathrm{K}$} & \multicolumn{2}{|c|}{$\delta_{\mathrm{MI}} \mathrm{cm}$} \\
\hline & Min. & Max. & Min. & Max. & & Mesuréc & Calculée & Adoptée & & & Min. & Max, \\
\hline \multirow{2}{*}{$\begin{array}{c}\mathrm{O} \dot{a} \\
200 \mathrm{~kg}\end{array}$} & \multirow[b]{2}{*}{5} & \multirow[b]{2}{*}{200} & \multirow[b]{2}{*}{16} & \multirow[b]{2}{*}{52} & \multirow[b]{2}{*}{32} & $\begin{array}{l}x_{1}=6.600 \\
x_{2}=4.100\end{array}$ & & 5.350 & 0,25 & 8,3 & 1,32 & 4,3 \\
\hline & & & & & & $x_{3}=2.340$ & & 2.340 & 0,57 & 7,2 & 1,15 & 3,74 \\
\hline \multirow{2}{*}{$\begin{array}{c}200 \mathrm{~kg} \\
\dot{\mathrm{a}} \\
2 \mathrm{t}\end{array}$} & \multirow{2}{*}{200} & \multirow{2}{*}{2.000} & \multirow{2}{*}{52} & \multirow{2}{*}{117} & \multirow{2}{*}{32} & $\begin{array}{l}x_{1}=400 \\
x_{2}=400\end{array}$ & $400+5.350 \times(52 / 16)$ & 18.000 & 7,6 & 5 & 2,6 & 5,6 \\
\hline & & & & & & $\begin{array}{l}x_{3}=1.140 \\
x_{4}=700\end{array}$ & $\begin{array}{l}1.140+2.340 \times(52 / 16) \\
700\end{array}$ & 7.500 & 18,4 & 3 & 1,56 & 3,4 \\
\hline \multirow{3}{*}{$\begin{array}{l}2 \mathrm{t} \\
\dot{a} \\
5 \mathrm{t}\end{array}$} & \multirow{3}{*}{2.000} & \multirow{3}{*}{5.000} & \multirow{3}{*}{117} & \multirow{3}{*}{153} & \multirow{3}{*}{32} & $\begin{array}{l}x_{1}=0 \\
x_{2}=1.060\end{array}$ & $530+5.350 \times(117 / 16)$ & 38.000 & 12,1 & 4,6 & 5,2 & 7 \\
\hline & & & & & & $\begin{array}{l}x_{3}=1.320 \\
x_{4}=1.860\end{array}$ & $1.540+2.430 \times(117 / 16)$ & 18.000 & 25,5 & 3,8 & 4,3 & 5,8 \\
\hline & & & & & & $x_{5}=2.460$ & & 2.460 & 18,7 & 2,8 & 3,2 & 4,7 \\
\hline $\begin{array}{l}5 \mathrm{t} \\
\dot{\mathrm{a}} \\
15 \mathrm{t}\end{array}$ & 5.000 & 15.000 & 153 & 220 & 38 & $\begin{array}{l}x_{1}=0 \\
x_{2}=500\end{array}$ & $500+5.350 \times(153 / 16)$ & 53.000 & 50 & 3,5 & 3,5 & 7,7 \\
\hline
\end{tabular}

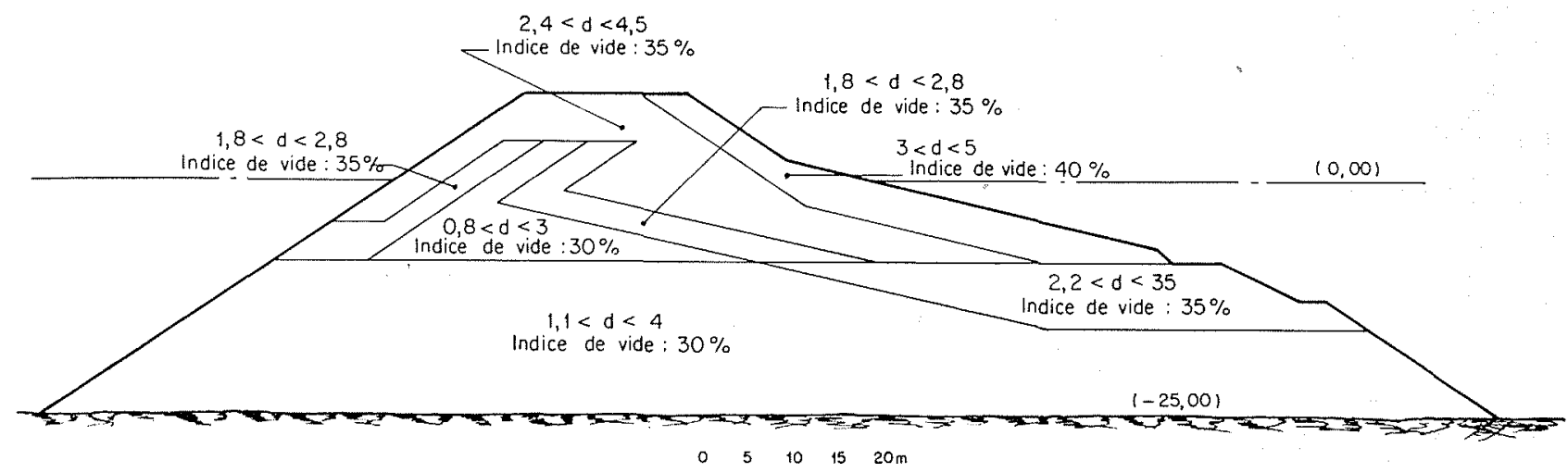

FIc. 24. - Plan du modèle réduit au $1 / 100$ d'une digue en enrochements avec masque étanche, la similitude étant respectée pour $2 a=10 \mathrm{~m}$. 
PROFIL $\mathrm{N}^{\circ} 4$

Profil en enrochements aVec masque étanche. - S Smilitude calculée pour $\mathrm{C}=2 a=10 \mathrm{~m}$

Echelle $1 / 10$

\begin{tabular}{|c|c|c|c|c|c|c|c|c|c|c|c|c|}
\hline \multirow{2}{*}{ Catégorie } & \multicolumn{2}{|c|}{$\begin{array}{l}\text { Poids } \mathrm{kg} \\
\text { densité } 2,8\end{array}$} & \multicolumn{2}{|c|}{$\begin{array}{c}\text { Granulométrie } \\
\delta \mathrm{cm}\end{array}$} & \multirow{2}{*}{$\begin{array}{c}\text { Indice } \\
\text { de } \\
\text { vide } \\
\varepsilon \%\end{array}$} & \multicolumn{3}{|c|}{ Largeur $x \mathrm{~cm}$} & \multirow{2}{*}{$\mathrm{C} \delta^{3} \varepsilon^{5} / x$} & \multirow{2}{*}{$\mathbf{K}$} & \multicolumn{2}{|c|}{$\begin{array}{c}\delta_{\text {modele }} \\
\mathrm{cm}\end{array}$} \\
\hline & Min. & Max. & Min. & $\operatorname{Max}$ & & Mesurée & Calculée & Adoptée & & & Min. & Max. \\
\hline \multirow{2}{*}{$500 \mathrm{~kg}$} & \multirow{2}{*}{10} & \multirow{2}{*}{500} & \multirow{2}{*}{20} & \multirow{2}{*}{74} & \multirow{2}{*}{30} & $\begin{array}{l}x_{1}=8.750 \\
x_{2}=6.100\end{array}$ & 7.000 & 7.000 & 3 & 5,5 & 1,10 & 4 \\
\hline & & & & & & $\begin{array}{c}x_{3}=2.800 \\
x_{4}=500\end{array}$ & 1.200 & 1.200 & 17 & 4 & 0,82 & 3 \\
\hline \multirow{2}{*}{$\begin{array}{l}500 \mathrm{~kg} \\
\dot{\mathrm{a}} \\
2 \mathrm{t}\end{array}$} & \multirow{2}{*}{500} & \multirow{2}{*}{2.000} & \multirow{2}{*}{74} & \multirow{2}{*}{117} & \multirow{2}{*}{35} & $\begin{array}{l}x_{1}=1.000 \\
x_{2}=1.500\end{array}$ & $7.000 \times(74 / 20)+1.500$ & 28.000 & 71 & 3 & 2,2 & 3,5 \\
\hline & & & & & & $\begin{array}{l}x_{3}=1.800 \\
x_{4}=800\end{array}$ & $1.200 \times(74 / 20)+1.300$ & 6.000 & 330 & 2,4 & 1,8 & 2,8 \\
\hline \multirow{2}{*}{$\begin{array}{c}2 t \\
\dot{a} \\
10 t\end{array}$} & \multirow{2}{*}{2.000} & \multirow{2}{*}{10.000} & \multirow{2}{*}{117} & \multirow{2}{*}{200} & \multirow{2}{*}{38} & $\begin{array}{l}x_{1}=1.000 \\
x_{2}=700\end{array}$ & $7.000 \times(117 / 20)+700$ & 40.000 & 200 & 2,2 & 2,6 & 4,4 \\
\hline & & & & & & $\begin{array}{l}x_{3}=900 \\
x_{4}=2.500\end{array}$ & $1.200 \times(117 / 20)+1.700$ & 8.500 & 940 & 2 & 2,35 & 4 \\
\hline \multirow{2}{*}{$\begin{array}{l}10 t \\
\dot{a} \\
20 t\end{array}$} & \multirow{2}{*}{10.000} & \multirow{2}{*}{20.000} & \multirow{2}{*}{200} & \multirow{2}{*}{252} & \multirow{2}{*}{40} & $\begin{array}{lr}x_{1}= & 0 \\
x_{2}= & 600\end{array}$ & $7.000 \times(100 / 20)+300$ & 70.000 & 1.100 & 1,8 & 3,6 & 4,5 \\
\hline & & & & & & $\begin{array}{l}x_{3}=600 \\
x_{4}=400\end{array}$ & $1.200 \times(2.000 / 20)+500$ & 20.000 & 4.000 & 1,3 & 2,6 & 1,95 \\
\hline
\end{tabular}

effectuées à l'aide d'une sonde à pointe vibrante qui supprime l'effet du ménisque capillaire.

Les résultats obtenus sont consignés sur les courbes ci-jointes. Nous donnons :

1. La valeur des coefficients de transmission pour différentes amplitudes et différentes périodes, en soulignant l'amplitude pour laquelle la similitude est théoriquement respectée (fig. 25). Nous avons transformé ces résultats dans le système d'axes coefficients de transmission-périodes, les courbes étant tracées à amplitude constante. Nous voyons facilement que la transmission est pratiquement négligeable pour les houles, mais dès que la période atteint 20 ou $30 \mathrm{~s}$, la transmission doit être prise en compte (fig. 25).
2. La valeur des coefficients de réflexion correspondants est donnée sur la planche 25 .

Nous retrouvons sur toutes ces courbes les lois qualitatives déjà énoncées lors̀ de l'étude des massifs de largeur constante :

a) l'effet de l'inertie se fait d'autant plus sentir que le rapport de la largeur de l'ouvrage sur la longueur d'onde est grand. Le profil avec blocs à contre-pente, moins large que le profil en enrochements, est donc relativement plus perméable aux ondes courtes que ce dernier;

b) le coeficient de transmission augmente lorsque l'amplitude décroît, par suite de la diminution des pertes par frottement;

c) la comparaison entre les courbes obtenues sur le même massif nature, mais calculées en 

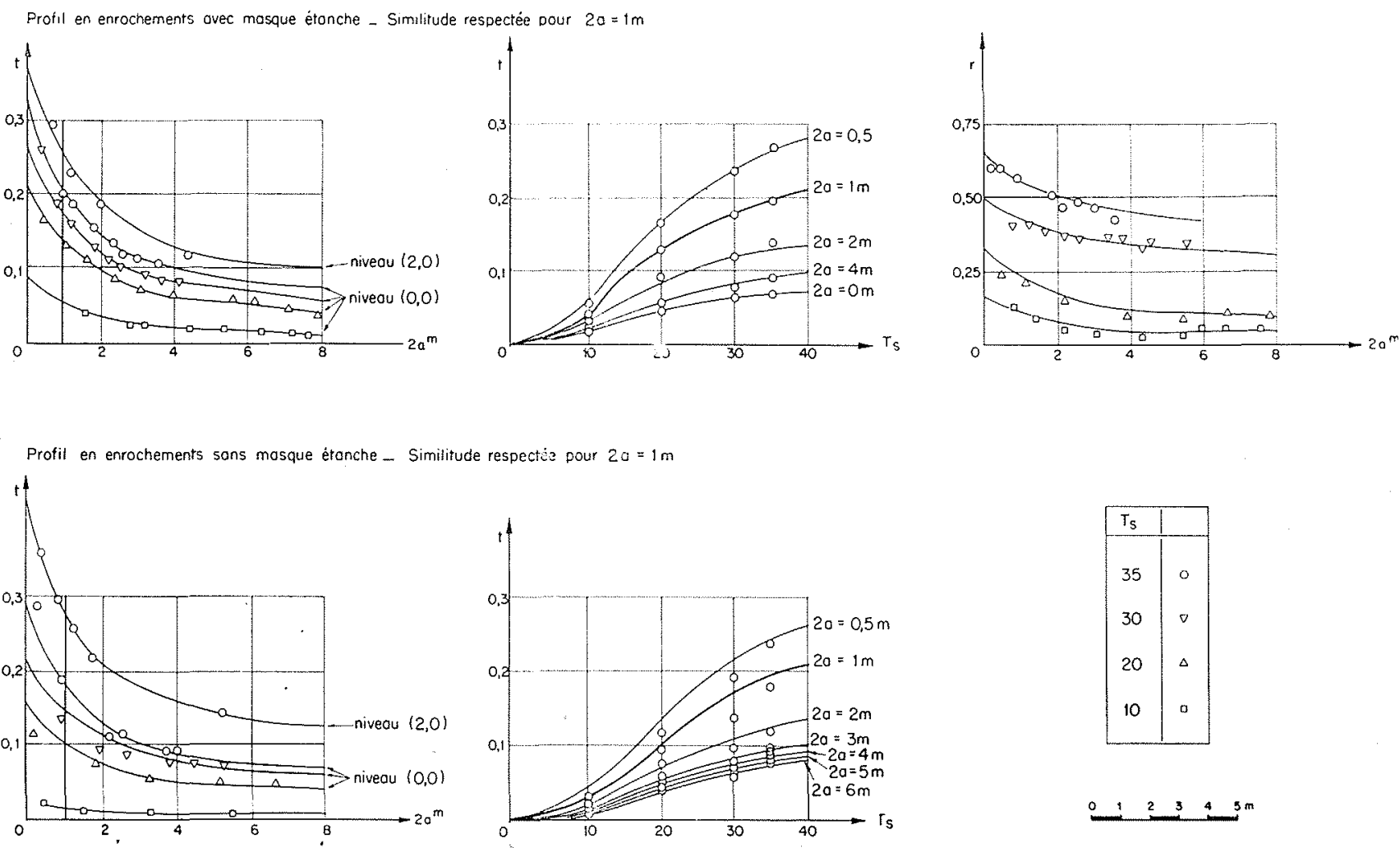

Profil avec blocs à contre-pente - Similitude respectée pour $2 a=1 m$
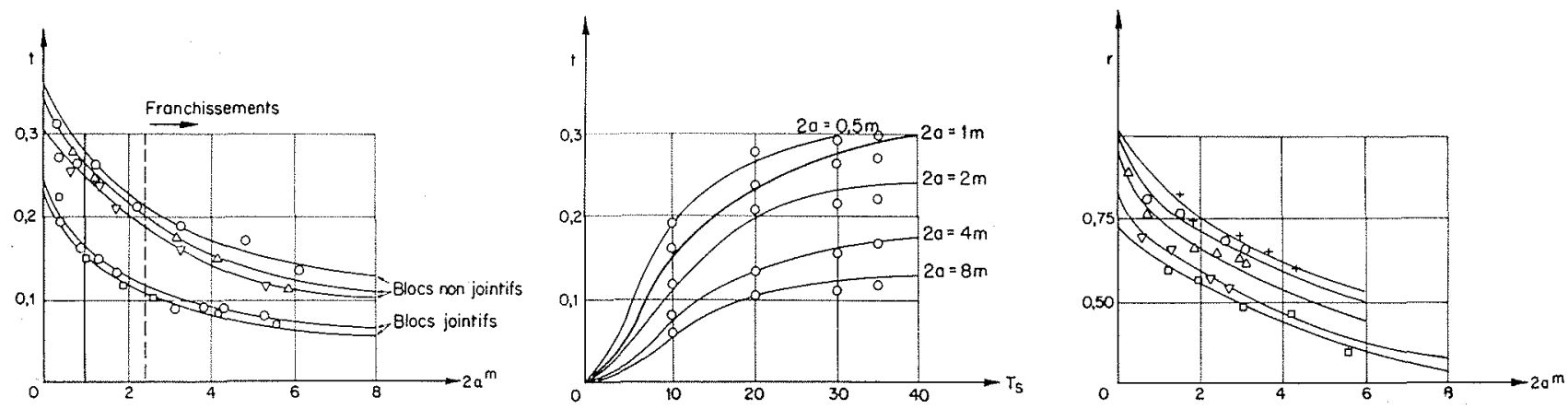

Profil en enrochements avec mosque étanche - Similitude respectée pour $20=10 \mathrm{~m}$
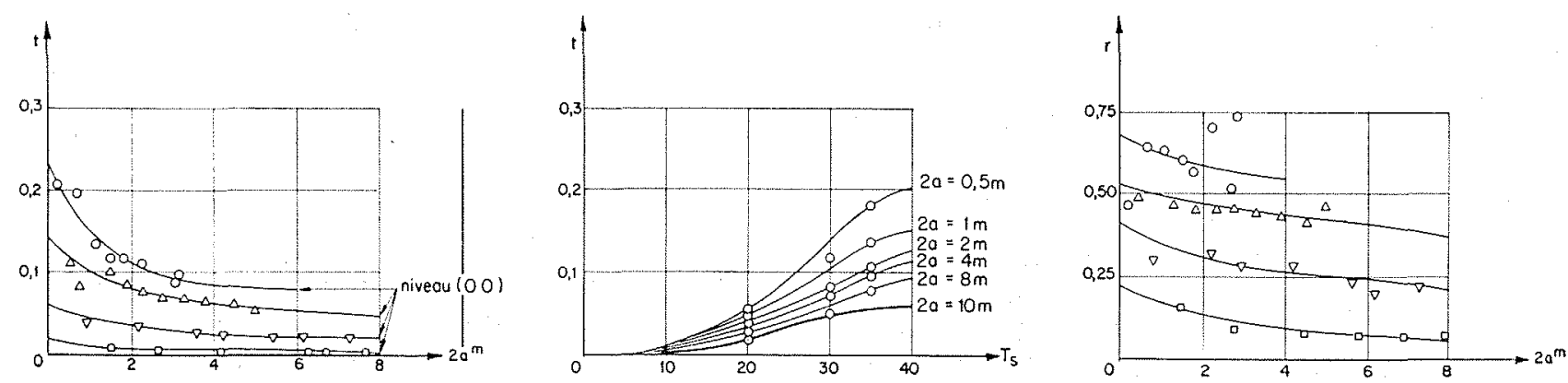

Fia. 25. - Résultats numériques des digues essayées. 
vue d'assurer la similitude pour des valeurs différentes de l'amplitude $(2 a=1 \mathrm{~m}$, $2 a=10 \mathrm{~m}$ ) montre l'importance de la turbulence induite par les houles accompagnant les ondes longues. La jetée est relativement moins perméable aux ondes longrues en période de tempête qu'en période de beau temps. Le coefficient de transmission réel en fonction de l'amplitude peut etre obtenu en interpolant les courbes expérimentales correspondant aux deux massifs calculés;

d) le coefficient de réflexion diminue avec la cambrure de la houle incidente, ce qui confirme les lois maintenant comnues de la réflexion;

c) la comparaison entre le massif en enrochements, avec et sans masque étanche, montre que ce dernier a une influence quasiment négliggeable. Ceci est dû au fait que ce masque étanche, construit dans la partie supérieure de la digue, ne fait pratiquement pas obstruction à la transmission des ondes longues. Les résultats obtenus ne semblent donc pas justifier la construction de ce masque tel que nous l'avons dessiné, c'est-à-dire en tout-venant de carrière;

f) par contre, la comparaison entre les coefficients de transmission obtenus sur le profil avec blocs à contre-pente jointifs et non-jo.ntifs montre l'importance de ce facteur, puisque l'écart de la transmission est de l'ordre de $30 \%$. On peut donc agir sur ces variables dans la mesure où la stabilité le permet et où cette modification n'augmente pas sensiblement le prix de la construction. (Pour éviter les sous-pressions sous les bloes de carapace, il est certain qu'il faut laisser entre les bloes une faible distance.)

\section{CHAPITRE VI}

\section{PRECISION DES RÉSULTATS}

Il existe trois sources d'erreurs :

1. Les erreurs sur la connaissance des lois de l'écoulement lorsque toutes les conditions naturelles sont parfaitement connues;

2. Les erreurs des mesures expérimentales;

3. Les erreurs sur la connaissance des conditions naturelles.

Nous ne ferons pas ici un calcul systématique de l'erreur probable. Cependant, nous pensons utile de s'attarder sur ce point, afin d'orienter les recherches futures.

\section{Erreur sur ta connaissance des lots de L'ÉCOULEMENT.}

Il est difficile de connaître l'erreur due à l'extrapolation des lois d'écoulement à travers des enrochements de granulométrie homogène d'un diametre inférieur à $20 \mathrm{~cm}$, aux lois de l'écoulement à traver's des enrochements de granulométrie hétérogène supérieure à $50 \mathrm{~cm}$. Notons toutefois que des essais nouveaux récemment effectuce au Laboratoire Dauphinois d'Hydraulique par M. Cohen de Lara (Cf. [24]) ont permis de repousser les limites de notre connaissance jusqu'à des nombres de Reynolds atteignant $10^{\circ}$. L'écoulement est alors pleinement turbulent et il semble logique d'extrapoler ces résultats aux cas qui nous intéressent.

Mais que faut-il entendre par connaissance des lois des écoulements? Nous connaissons les variables qui influent sur les pertes de charge et la forme de la relation qui les lie. Nous pouvons déterminer le coefficient $C$ et la fonction $f$ à l'aide des résultats obtenus, mais, malgré tout le soin apporté aux essais, l'erreur introduite dans ce coefficient est de l'ordre de $50 \%$. Ceci est principalement dû à la difficulté de préciser le diamètre caractéristique des enrochements et l'erreur sur la mesure de l'indice de vide.

De plus, le coefficient $\mathrm{C}$ est modifié par la rugosité des blocs d'une façon "qui est encore mal précisée.

De plus, le passage des lois du régime continu aux lois du régime périodique s'accompagne aussi d'une erreur; mais nous avons vu qu'à partir du moment où les couches limites turbulentes, se développant à partir de chaque enrochement, se joignent pour établir dans le massif une turbulence homogène, la notion de charge efficace pouvait être introduite avec succès, bien que la définition de la charge efficace puisse être modifiée quand il y a déferlement sur une paroi oblique. Dans l'état actuel de la technique, il est difficile de préciser dans quelle proportion. 


\section{ERrEURS EXPÉRIMENTALES.}

Les erreurs expérimentales sont principalement dues au manque de précision dans la réalisation des profils. Nous avons vu qu'il fallait à la fois réaliser des conditions hydrodynamiques et hydrostatiques, ce qui nécessite de doser par approximation des mélanges binaires ou ternaires. La recherche de la précision nécessiterait des efforts longs et coûteux, d'ailleurs injustifiés, car la principale source d'erreurs restera la connaissance des conditions naturelles.

\section{ERreutrs dans LA CONNAISSANCE DES CONDI- TIONS NATURELLES.}

Dans l'état actuel de la technique, les ressources du modèle réduit sont encore bien supérieures aux connaissances des phénomènes naturels. En effet, nous avons vu cuelle est l'importance de la détermination de la granulométrie caractéristique et de l'indice de vide sur la valeur du coefficient de transmission. Or, dans la nature, nous connaissons le diamètre caratéristique des enrochements de carrière avec une précision de l'ordre de $100 \%$. Quant à l'indice de vide, une erreur de $5 \%$ sur celui-ci est fort possible.

Il faudrait, pour réduire cette erreur, connaitre les courbes granulométriques des produits de carrière; or, généralement, l'étude des profils est faite avant leur exploitation et l'on sait qu'il est très difficile de prévoir ce que donnera une carrière.

Remarquons que les données basées sur l'analyse des produits de carrière est encore insuffisante, car il faut ajouter l'effet de tassement dans le temps, qui reste une variable inconnue, le développement des algues, l'introduction en période de beau temps de sable à travers le noyau de tout-venant, etc.

$$
\text { * }
$$

Pour effectuer le calcul d'erreurs, il y a lieu de distinguer les erreurs par rapport à la nature et les erreurs relatives entre modèles, qui sont généralement beaucoup plus faibles. Or, les résultats des différents modèles construits à la même échelle, suivant les mêmes principes, permettent des comparaisons qui, à elles seules, justifient en bonne part les essais.

Les erreur's possibles énumérées au début de ce chapitre sont réduites à quelques erreurs fondamentales. En effet, de nombreux phénomènes plus ou moins connus se retrouvent intégralement sur le modèle d'une façon quasi identique à celle de la nature : déferlement, absorption d'énergie à la paroi, etc. L'erreur sur la connaissance exacte des lois de l'écou. lement se limite donc à la détermination de l'erreur qui en résulte dans l'établissement des lois de similitude, c'est-à-dire l'erreur faite sur l'énergie perdue dans le massif.

Les erreurs de mesures expérimentales sont faibles et peuvent être en général négligées. Les erreurs essentielles restent celles sur la connaissance des conditions naturelles.

Pour faire le calcul d'erreur relatif à nos essais sur les modèles de digues, nous devons faire le bilan de l'énergie de l'onde arrivant sur l'ouvrage :

-. l'énergie incidente $\mathbf{E}_{i}$ se répartit en énergie absorbée dans le déferlement sur la paroi : $\mathrm{E}_{l l}$,

- en énergie réfléchie : $\mathrm{E}_{i}$,

- en énergie absorbée par frottement à l'intérieur de la digue: $\mathrm{E}_{\iota,}$

- et enfin en énergie transmise : $\mathrm{E}_{t}$; ce qui se traduit par l'équation :

$$
\mathrm{E}_{i}=\mathrm{E}_{t l}+\mathrm{E}_{r}+\mathrm{E}_{t}+\mathrm{E}_{t}
$$

L'erreur sur l'énergie transmise $\mathrm{E}_{t}$ est donc égale à la somme des erreurs faites sur les autres termes, c'est-à-dire :

$$
\Delta \mathrm{E}_{t}=\Delta \mathrm{E}_{l}+\Delta \mathrm{E}_{r}+\Delta \mathrm{E}_{a}+\Delta \mathrm{E}_{i}
$$

Or, les erreurs faites sur l'énergie incidente, sur l'énergie absorbée dans le déferlement et sur l'énergie réfléchie (phénomènes qui répondent parfaitement à la similitude de Froude) sont tout à fait négligeables. (On a mesuré que ces phénomènes peuvent présenter un léger effet d'échelle lorsque celle-ci est de l'ordre du $\left.1 / 150^{\circ}\right)$

On peut donc écrire que l'erreur faite sur l'énergie transmise est égale à l'erreur faite sur l'énergie absorbée dans le massif :

$$
\Delta \mathrm{E}_{t} \# \Delta \mathrm{E}_{a}
$$

L'énergie absorbée dans le massif par unité de temps est de la forme : $\mathrm{RU}^{2}$.

Or,

$$
\mathrm{RU}=\frac{g \Delta \mathrm{H}}{\Delta l} \frac{\mathrm{C}_{i}(\mathrm{U} d / \mathrm{v})}{\varepsilon^{5}} \frac{\mathrm{U}^{2}}{2 d}
$$

de telle sorte que l'énergie absorbée prend la forme :

$$
\mathrm{E}_{a} \cong\left(\frac{g \mathrm{H}_{e}}{l}\right)^{3 / 2} \sqrt{\frac{2 d \varepsilon^{5}}{\mathrm{C}(\mathrm{Ud} / \nu)}}
$$

et l'erreur maximum faite sur $\mathrm{E}_{a}$, donc sur $\mathrm{E}_{t}$, est égale à :

$$
\begin{aligned}
& \frac{\Delta \mathrm{E}_{t}}{\mathrm{E}_{a}}=\frac{\Delta \mathrm{E}_{a}}{\mathrm{E}_{a}}=\frac{3}{2} \frac{\Delta \mathrm{H}_{e}}{\mathrm{H}_{e}}+ \frac{3}{2} \frac{\Delta l}{l}+\frac{1}{2} \frac{\Delta d}{d} \\
&+\frac{5}{2} \frac{\Delta \varepsilon}{\varepsilon}+\frac{1}{2} \frac{\Delta \mathrm{C}}{\mathrm{C}}
\end{aligned}
$$


Appelons $N$ cetle expression. L'erreur quadratique moyenne (erreur probable), que nous appellerons $M$, sera égale à :

$$
\frac{\Delta^{\prime} \mathrm{E}_{t}}{\mathrm{E}_{a}}=\frac{\Delta^{\prime} \mathrm{E}_{l}}{\mathrm{E}_{t}}=\sqrt{\frac{3}{2}\left(\frac{\Delta \mathrm{H}_{l}}{\mathrm{H}_{c}}\right)^{2}+\frac{3}{2}\left(\frac{\Delta l}{l}\right)^{2}+\left(\frac{1}{2} \frac{\Delta d}{d}\right)^{2}+\left(\frac{5}{2} \frac{\Delta \varepsilon}{\varepsilon}\right)^{2}+\left(\frac{1}{2} \frac{\Delta \mathrm{C}}{\mathrm{C}}\right)^{2}}
$$

L'erreur relative à l'énergie incidente est donc égale à :

$$
\frac{\Delta \mathrm{E}_{t} \text { ou } \Delta^{\prime} \mathrm{E}_{t}}{\mathrm{E}_{i}}=\frac{\mathrm{E}_{t}}{\mathrm{E}_{i}}(\mathrm{~N} \text { ou } \mathrm{M})
$$

Il est difficile d'évaluer $\mathrm{E}_{a}$, mais on connaît l'ordre de grandeur de l'énergie perdue dans le déferlement $\mathrm{E}_{l l}$ et l'énergie réfléchie $\mathrm{E}_{r}$. D'autre part, on peut négliger l'énergie transmise devant $\mathrm{E}_{l l}$ on $\mathrm{E}_{i}$, de telle sorte que l'erreur $\Delta \mathrm{E}_{l} / \mathrm{E}_{i}$ devient égale à :

$$
\frac{\Delta \mathrm{E}_{t} \text { ou } \Delta^{\prime} \mathrm{E}_{t}}{\mathrm{E}_{i}}=\left[1-\frac{\mathrm{E}_{t}+\mathrm{E}_{r}}{\mathrm{E}_{i}}\right](\mathrm{N} \text { ou } \mathrm{M})
$$

L'erreur relative faite sur le coefficient de transmission $t$ est évidemment plus forte puisqu'elle ne dépend plus directement de l'énergie absorbée dans le déferlement et de l'énergie réfléchie. En effet, on a :

$$
\frac{\Delta \mathrm{E}_{7} \text { ou } \Delta^{\prime} \mathrm{E}_{t}}{\mathrm{E}_{t}}=\frac{\mathrm{E}_{c}}{\mathrm{E}_{l}}(\mathbf{N} \text { ou } \mathrm{M})
$$

d'oì:

$$
\frac{\Delta t \text { on } \Delta^{\prime} t}{t}=\frac{1}{2} \frac{\mathrm{E}_{t !}}{\mathrm{E}_{t}}(\mathrm{~N} \text { on } \mathrm{M})
$$

Nous appliquerons ces formules en tenant compte des remarques faites ci-dessus et nous distinguerons les deux cas : erreurs faites entre modèle et nature et erreurs faites entre modèles.

Dans le premier cas, nous avons vu que l'erreur faite sur $\mathrm{C}(\mathrm{U} d / \mathrm{v})$ peut être de $50 \%$ (cette erreur englobant l'erreur due au nombre de Reynolds), celle sur $d$ de $100 \%$ et celle sur $\varepsilon$ de $5 \%$.

On peut évaluer l'erreur faite sur $\mathrm{H}_{e}$ à $33 \%$ et celle faite sur le calcul de $l$ à $33 \%$. Notons bien que ces erreurs ne sont commises que gans la détermination du coefficient de frottement, donc du grossissement $K$ du matériau. Il est évident que l'erreur faite sur la largeur $l$ dans la réalisation du modèle est tout à fait négligeable, de même que l'erreur faite penm dant l'essai sur la charge efficace $H_{e}$ puisque l'on reproduit presque parfaitement la houle incidente et les phénomènes sur le parement de la digue.

Remarquons en toute rigueur que notre calcul d'erreurs n'est valable que si les erreurs relatives sur chaque terme restent faibles, ce qui n'est évidemment pas toujours le cas. Cependant, nous appliquerons les méthodes classiques qui nous donnent une idée suffisante de la précision globale.

Les expressions $N$ et $M$ sont alor's égales, tout calcul fait :

$$
\begin{aligned}
& N=1,875 \\
& M=0,820
\end{aligned}
$$

Si l'on considère que l'énergie relative perdue dans le déferlement $\mathrm{E}_{a} / \mathrm{E}_{i}=0,5$ et l'énergie réfléchie $\mathrm{E}_{r} / \mathrm{E}_{i}=0,3$, on en déduit que l'erreur maximum de l'énergie transmise relative à l'énergie incidente est égale à :

$$
\Delta \mathrm{E}_{t} / \mathrm{E}_{i}=0,2 \times 1,875=37,50 \%
$$

et l'erreur probable :

$$
\Delta^{\prime} \mathrm{E}_{t} / \mathrm{E}_{i}=0,2 \times 0,82=16,40 \%
$$

Maintenant, si l'on considère que l'énergie absorbée dans la digue est égale à l'énergie transmise, l'erreur maximum faite sur $t$ :

$$
\Delta t / l=0,5(1,875)=93,7 \%
$$

et l'erreur probable:

$$
\Delta^{\prime} t / t=0,5 \times 0,82=41 \%
$$

Examinons maintenant les erreurs relatives entre modèles qui sont beaucoup plus faibles.

L'erreur faite sur $d$ est uniquement due aux différences apportées dans la réalisation des modèles : elle dépend du dosage des mélanges et de leur tassement; elle n'excède donc pas $10 \%$; il en est de même de l'erreur faite sur $\varepsilon$, que l'on peut évaluer à $2 \%$.

L'erreur commise sur la charge efficace $\mathrm{H}_{\epsilon}$ est nulle si le profil extérieur eşt géométriquement semblable à celui de la nature puisque les coefficients $K$ sont calculés pour la même amplitude.

Si le profil extérieur n'est pas le même (pente différente causée par distorsion), il s'ensuit une légère erreur dans l'appréciation de la différence des conditions à la paroi. Dans ce cas :

$$
\frac{\Delta \mathrm{H}_{e}}{\mathrm{H}_{e}}=\frac{\Delta r}{1+r}
$$

Si on évalue $\Delta r / r$ à $0,1, r$ à 0,5 :

$$
\frac{\Delta \mathrm{H}_{c}}{\mathrm{H}_{c}}=\frac{0,05}{1,5}=3 \%
$$

L'erreur commise sur la largeur $l$ est quasi- 
ment négligeable quand on passe d'un profil à l'autre.

L'erreur commise sur C n'est plus due à une connaissance imparfaite des lois de l'écoulement puisque l'on travaille toujours sensiblement dans les mèmes conditions de turbulence (et à ce propos, il y a intérêt à comparer des modèles exécutés à la même ćchelle), mais aux différences dans l'appréciation des nombres de Reynolds entre les deux modèles.

Si l'on considère que sur le modèle le régime est toujours laminaire:

$$
\begin{gathered}
\mathrm{C}=\frac{\mathrm{C}+\mathrm{U}}{\mathrm{U} d} \\
\frac{\Delta \mathrm{C}}{\mathrm{C}}=\frac{\Delta \mathrm{U}}{\mathrm{U}}+\frac{\Delta d}{d}
\end{gathered}
$$

et l'on peut évaluer l'erreur maximum $\Delta C_{/} / \mathrm{C}$ à 0,2 et l'erreur quadratique $\Delta \mathrm{C} / \mathrm{C}$ à 0,14 .

Finalement, dans ce cas, rès termes $\mathrm{N}$ et $\mathrm{M}$ deviennent, tous calculs. faits :

$$
\begin{aligned}
& \mathrm{N}=0,7 \mathrm{~s} \\
& \mathrm{M}=0,30
\end{aligned}
$$

Et l'on en déduit, dans les mèmes conditions déjà traitées, les erreurs maximums:

$$
\begin{aligned}
& \frac{\Delta \mathrm{E}_{t}}{\mathrm{E}_{i}}=0,78 \times 0,2=15,60 \% \\
& \frac{\Delta t}{t}=\frac{1}{2} \quad 0,78=39 \%
\end{aligned}
$$

et les erreurs probables:

$$
\frac{\Delta \mathrm{E}_{t}}{\mathrm{E}_{i}}=0,2 \times 0,30=6 \%
$$

et :

$$
\frac{\Delta^{\prime} t}{t}=\frac{1}{2} \times 0,30=15 \%
$$

Sans doute faudrait-il alors ajouter les erreurs fortuites dues aux mesures expérimentales qui, cette fois, ne sont plus négligeables devant les autres termes.

\section{Conclusion.}

Le choix définitif du profil d'une digue en enrochements dépend des données économiques et techniques.

Ces dernières sont généralement fonction des possibilités des carrières aux environs du port, des moyens de levage de l'entreprise qui exécute le projet, des conditions d'exploitation du port et des conditions de stabilité aux houles de tempètes.

On ne se préoccupe généralement de la perméabilité que lorsque les houles se transmettent a travers la digue d'une façon gênante pour les usagers du port.

Le probleme de la perméabilité des digues en enrochements peut maintenant ètre étudié avant lear réalisation, avec une précision suffisante pour les besoins de la technique.

Ce probleme a son importance, non pas tant pour les houles, car on sait facilement s'en protéger, que pour les ondes de seiches qui se transmettent avec une grande facilité. Nous traiterons par ailleurs ce problème qui déborde le cadre de notre ouvrage, mais nous insisterons simplement sur son importance, des que l'on craint l'effet de seiches portuaires $\left({ }^{\star}\right)$.

En conséquence, sans avoir résolu complètement le problème de la perméabilité des digues, nous espérons du moins avoir contribué à le résoudre et à en avoir examiné tous les aspects.

Il restera aux futures recherches d̀ préciser l'influence de certaines variables pour lesquelles nos théories et nos essais n'ont pas apporté une complete solution. Il serait souhaitable, dans ce sens, de préciser l'influence de l'indice de vide, de l'effet de tassement dans le temps, etc.

Enfin, il serait utile d'analyser avec soin les caractéristiques des digues réalisées, afin de baser les futures recherches théoriques et expérimentales sur des données sûres. C'est seulement quand ces donnces seront obtenues qu'il sera rentable d'effectuer une analyse peut-être plas poussée que celle-ci. Notons cependant que les r'ésultats présentés suffisent pour les besoins actuels de la technique portuaire.

(*) Pour résoudre le problème des seiches portuaires, nous ne voulons pas dire qu'il faut imperméabiliser les digues en enrochements, bien au contraire; le probleme est trop complexe pour être analyse ici, aussi renvoyonsnous le lecteur à une prochaine publication. 


\section{B I BLIOGRA PH IE}

1. Kर. Trnzaghi, - Eng. Nems Record 95, 1925, p. 382.

2. H. E. Rose. - Fluid Flow through beds of granular materials Some aspects of fluid flow. Edeward Arnold and Co., London, 1951, p. 136.

3. H. LaFennene. - Annales du Ministère de l'Agriculture, 1938 , fas. 67, p. 51

4. L. Escande. - Proceedings Minnesota International Hydraulics Convention, A.I.R.H., sept. 1953 , p. 547 .

5. M. Muskit. - The flow of homogeneous fluids through porous media McGraw Hill, 1937, p. 763 .

6. G. Cohre de LaRA. - La Hollille Blanche, $\mathrm{n}^{\circ} 2$, mai 1953, p. 167.

7. G. Schnebueli. - La Houille Blanche, mars-avril 1955, p. 111

8. J. Valensi et G. Clarion. - Bulletin de la Société Française des Mécaniciens, $n^{\circ} 8,2$ trimestres 1953.

9. J. Yalensi et C. Chamion. - Comptes rendus de l'Académie des Sciences, 1953, t. 237, $n^{\circ} 19$, p. 1138.

10. J. Vatemisors. - Contribution à l'étude de la mesure des pressions variables. Herman et $C_{*}^{\circ}, 1948$.

11. F. Crausse. - Publications Serv. Tech. du Ministere de l'Air, 95, p. 42.

12. F. Birsel. - La Houille Blanche, $\mathrm{n}^{\circ} 2$, marsavril 1950, p. 157 .

13. T. H. Haverock. -- Forced, surfaces waves on water, Philosophical Magazine, série 7, XVIII, 1929, 1. 569 .
14. F. Bresei. -. La Houille Blanche, 1951, p. 975.

15. J. Rnavtcheniso. - Proceedings of the fifth conference on Coastal Engineering, sept. 1954, p. 50.

16. F. Buesel ET B. LE Méhautó. -- La Houlle Blanche, mars-avril 1955 , p. 130.

17. L. Greslou ET Y. MarÉ. - Proceedings of the fifth conference on Coastal Engineering, sept. 1954, p. 68 .

18. A. Wabret. - Communication à la Société Hydrotechnique de France, juin 1951.

19. B. LF MÉnaúé. - Le filtre a houle progressif, La Honille Blanche, $\mathrm{n}^{\circ} 3,1955$, p. 4 .

20. J. Boudan. - La Honille Blanche, $\mathrm{n}^{\circ} 4$, aoûtsept. 1953 , p. 526 .

21. J. VAlambois. - Communication à la Société Hydrotechnique de France, 19-5-1952, p. 1 .

22. B. LE Méhauté. - Comptes rendus de l'Académie des Sciences, juillet 1957, p. 276.

23. B. LE MhHatś. - Communication à la Société Hydrotechnique de France. IV $V^{\text {s }}$ Journées de l'Hydralique, Paris, 1956, Question III, Rapport $\mathrm{n}^{\circ} 4$

24. COHEN DE LARA. - Communication à la Société Hydrotechnique de France. $I V^{c s}$ Journées de l'Hydranlique, Paris, juin 1956, Question IV, Rapport $n^{\circ} 7$.

25. E. O. Macagio. - La Houille Blanche, no 1, janv.fév. 1954 , p. 10 .

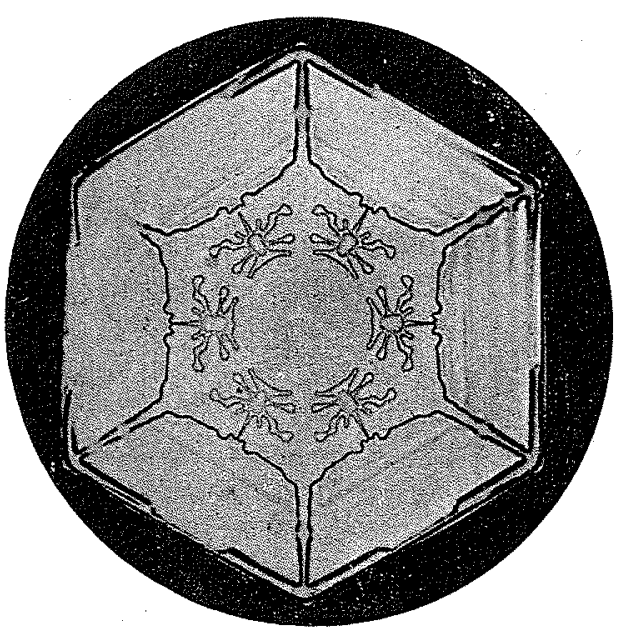

